\title{
Liame
}

Liame Histoire et histoire de l'art des époques moderne et contemporaine de l'Europe méditerranéenne et de ses périphéries

\section{La monarchie absolue pendant les deux dernières décennies de l'Ancien Régime : les assemblées provinciales de Necker, Calonne et Loménie de Brienne}

\section{Stephen Miller}

\section{(2) OpenEdition}

\section{Journals}

\section{Édition électronique}

URL : http://journals.openedition.org/liame/97

DOI : 10.4000/liame.97

ISSN : 2264-623X

Éditeur

CRISES - Centre de Recherches Interdisciplinaires en Sciences Humaines et Sociales de Montpellier

\section{Référence électronique}

Stephen Miller, «La monarchie absolue pendant les deux dernières décennies de l'Ancien Régime : les assemblées provinciales de Necker, Calonne et Loménie de Brienne», Liame [En ligne], 23 | 2011, mis en ligne le 26 octobre 2011, consulté le 20 avril 2019. URL : http://journals.openedition.org/liame/97 DOI : 10.4000/liame.97

Ce document a été généré automatiquement le 20 avril 2019. 


\title{
La monarchie absolue pendant les deux dernières décennies de l'Ancien Régime : les assemblées provinciales de Necker, Calonne et Loménie de Brienne
}

\author{
Stephen Miller
}

En 1778 et 1779, Jacques Necker, directeur général des finances, créa des assemblées dans le Berry et en Haute-Guyenne. Le roi designa seize députés, dont quatre siégèrent pour le clergé, quatre pour la noblesse, et huit pour le tiers-état, puis ces députés en nommèrent trente-deux autres dans la même proportion, pour discuter et voter ensemble au sujet de la répartition de l'impôt entre les paroisses et contribuables, de l'administration des travaux publics, et de toute autre réforme qu'ils pourraient proposer.

En 1787, le contrôleur général Calonne présenta le projet plus ambitieux de créer des assemblées dans tous les pays d'élection (dénués d'états provinciaux), soit une zone couvrant plus de la moitié du royaume. Ces assemblées devaient être complétées par des assemblées de district (élections) et des conseils de paroisse. Le recrutement de ces assemblées devait être basé sur la possession de la propriété plutôt que sur l'appartenance à un état. De plus, les paroissiens satisfaisant les critères de propriété devaient élire un conseil d'administrateurs, pour siéger avec le curé sous la présidence du seigneur.

La monarchie nomma les premiers députés des assemblées provinciales, puis ceux-ci nommèrent le reste de leurs membres ainsi que les premiers membres des assemblées de district. Ces membres nommèrent à leur tour le reste de leurs assemblées. Dans les années suivantes, les membres élus des conseils de paroisse devaient renouveler les membres des assemblées de district qui, ensuite, devaient faire de même pour les assemblées provinciales. 
Alexis de Tocqueville estima que les assemblées provinciales testèrent les principes même de la monarchie et eurent des conséquences profondes pour la stabilité du régime. L'image de la symétrie administrative - affirma Tocqueville - avait tellement imprégné la pensée des ministres royaux qu'ils mirent en place simultanément une série homogène d'assemblées sur tout le territoire sans égard pour les coutumes historiques des provinces. Puisque les Français avaient développé une dépendance à l'Etat pour tout ce qui pourrait assurer leur tranquillité et leur bien-être matériel, cette réforme radicale de l'administration aurait perturbé leurs affaires privées et aurait fourni une des causes immédiates de la Révolution ${ }^{1}$.

Cette interprétation a été écartée au XXe siècle par les recherches de Pierre Renouvin et de Maurice Bordes. Ces historiens ont soutenu que la monarchie préserva la division des états et donna aux privilégiés une prépondérance d'influence dans les assemblées à cause de l'opposition des parlements, du haut clergé et de la haute noblesse. En outre, la monarchie priva les assemblées de fonds et permit aux intendants de garder la mainmise sur l'administration. Dépourvues d'initiative et dominées par les privilégiés, les assemblées ne réalisèrent que des projets négligeables et n'élargirent pas les bases de leur recrutement $^{2}$.

Néanmoins, Peter Jones, le dernier historien à avoir réalisé des recherches sur ces assemblées, a ranimé l'interprétation tocquevillienne sur le thème de "l'absolutisme réformateur ", caracterisé par des intendants désireux de procéder à des améliorations et par un personnel professionnel et bureaucratique dans le domaine des finances. Les assemblées provinciales - soutient Jones - furent la réforme la plus lourde de conséquences dans le sens où les élections villageoises fournirent un apprentissage de la participation politique et convainquirent certains contemporains d'une transition vers une monarchie constitutionnelle. En demandant aux membres des assemblées de réfléchir aux abus du régime, la monarchie amena ses sujets à voir les problèmes posés par les privilèges et à rejeter une société divisée en ordres politiques ${ }^{3}$.

P. Jones a raison de souligner l'impulsion réformatrice de la monarchie. Je soutiens que les ministres royaux, les grands nobles et leurs secrétaires - les auteurs des projets d'assemblées - avaient discerné avec clarté les problèmes de la monarchie absolue d'une manière semblable à celle des historiens actuels et proposèrent des réformes radicales pour la renouveler. Pourtant, si l'on se focalise sur ces projets d'assemblées, on ignore toutes les preuves d'opposition.

Dans ce qui suit, j'examine cette opposition et je démontre que l'on peut sonder plus intimement les fondements de l'Etat et de la société que ne l'ont fait P. Renouvin et M. Bordes, dont les écrits soulèvent autant de questions qu'ils en résolvent. P. Renouvin et M. Bordes n'ont pas examiné pourquoi certains nobles se sont opposés à la représentation politique et pourquoi certains nobles n'ont pas voulu faciliter ces réformes de grande envergure. Pourquoi la couronne avait-elle tant de difficultés à triompher de l'opposition ? Quels furent les éléments au sein de l'Etat qui freinèrent l'activité des assemblées et soutinrent le pouvoir des intendants ? Dans le but de répondre à ces questions, je vais considérer l'un des aspects de l'opposition aux assemblées, à savoir les attitudes et les intérêts du roi, des princes, des grands nobles de la cour et les alliances qu'ils forgèrent dans les provinces en faveur de l'absolutisme traditionnel.

Les cours souveraines s'étaient opposées plusieurs fois aux efforts produits par la monarchie pour augmenter certains impôts depuis le milieu du XVIII ${ }^{e}$ siècle. Elles contestaient les procédés arbitraires des ministres. Ceux-ci conclurent que le meilleur 
moyen de mettre les finances sur des bases plus sûres était d'offrir de la représentation politique aux propriétaires. Pour développer des projets concrets d'assemblées, ils s'appuyèrent sur un demi-siècle d'écrits de ministres, d'agents et officiers du roi. Il est intéressant de remarquer qu'en présentant les mérites des assemblées, ces auteurs presque tous de la haute noblesse - décrivait la monarchie de la même manière que le font nos historiens contemporains, tels que William Beik, Marie-Laure Legay, Robert Descimon et Christian Jouhaud. Selon cette interprétation de la monarchie absolue, une interprétation prédominante en ce moment, le roi s'était imposé, non pas en réduisant les droits des nobles, ni en créant une administration uniforme et rationnelle du royaume, mais plutôt en intégrant à l'Etat les droits traditionnels et autonomes des nobles, de même que les droits à prélever des commissions en tant que financiers, de percevoir des rentes en tant que seigneurs et prélats, et de jouir des composantes du régime en tant que juges et administrateurs vénaux ${ }^{4}$.

Pierre Samuel du Pont de Nemours - s'inspirant d'un demi-siècle d'écrits sur les assemblées, sous la plume des marquis d'Argenson et de Mirabeau, ainsi que de L'Averdy, de Chaumont de la Galaizière et de Le Trosne - rédigea d'abord un mémoire sur les municipalités pour Turgot, en 1775, puis le projet d'assemblées de Calonne, en 1787. Dans le mémoire de 1775 , il soutenait que le roi et ses ministres avaient reçu une éducation religieuse et dépassée, qu'ils avaient tiré leurs solutions des problèmes de l'Etat dans des époques passées d'ignorance, et que, par conséquent, ils avaient eu des embarras face aux privilégiés. Les usages traditionnels du gouvernement, soutenait du Pont de Nemours, - à savoir l'accaparement des fonctions publiques en tant que propriété privée - devaient être remplacés par un ordre constitutionnel d'assemblées provinciales gouvernées par les mêmes lois dans tout le territoire, pour que le roi puisse résoudre les problèmes grâce à une législation générale plutôt qu'à des avis particuliers offerts à lui sur chaque affaire distincte. Car les contribuables considéraient les commissionaires et contrôleurs fiscaux du roi comme des agents étrangers d'un gouvernement envahissant. Si des assemblées de contribuables, affirmait du Pont de Nemours, avaient la responsabilité de la répartition de l'impôt et d'entendre les réclamations, les sujets du roi reconnaîtraient leurs intérêts communs avec le roi et seraient plus à même de payer leurs impôts 5 .

Dans les justifications de sa politique, Jacques Necker critiquait l'usage traditionnel et inhabile qui consistait à céder les fonctions publiques à des officiers à la recherche d'un gain personnel au lieu de s'occuper de l'exécution efficace des affaires publiques. "Subdélégues, officiers d'élections, directeurs, receveurs \& contrôleurs des vingtièmes, [...] officiers des gabelles, [...] tous [...] assujettissent à leur petite autorité [...] des contribuables ignorants. [...] Si d'un pareil chaos il peut enfin sortir un système simple \& régulier [...] on ne peut l'espérer [...] qu'à l'aide des [assemblées provinciales] ${ }^{6} »$. Celles-ci auraient été susceptibles d'une surveillance ministerielle et aussi de mériter la bonne volonté des contribuables. Le succès des assemblées du Berry et de la Haute-Guyenne, écrivait Necker, avait résulté de la publication de leurs procès-verbaux. Cette publicité avait aidé les assemblées à gagner l'appui de l'opinion publique et ainsi à exécuter des décisions politiques opposées aux pratiques traditionnelles?

Tout ceci pour dire qu'il y avait des ministres et des grands nobles qui cherchaient à changer les bases sociales de la monarchie. Cependant, ils ne réussirent pas à réaliser leurs projets à cause de l'opposition qu'ils soulevaient, dont une bonne partie coexistait avec eux au sein de l'Etat. Les études sur les assemblées provinciales, et sur les réformes royales dans l'ensemble, n'ont pas prêté attention au patrimoine féodal du roi, des 
princes, et des grands nobles de la cour. Or, depuis le début du XVII ${ }^{e}$ siècle et jusqu'en 1789 , le roi a régulièrement revendiqué son droit féodal en vertu de la directe universelle, autrement dit que tous les fiefs appartenaient au roi. Le roi et ses ministres avaient l'intention d'obliger les nobles à lui offrir des paiements au lieu de lui devoir des charges féodales ${ }^{8}$.

Le roi fit aussi respecter ses droits pour l'avantage de sa famille, dont les membres imitèrent le roi en mettant en valeur leurs seigneuries. Le duc d'Orléans jouissait de revenus annuels de sept millions de livres, les plus élevés du royaume, dont plus de la moitié était tirée de l'apanage fourni par le roi. Les deux tiers du revenu du fief provenaient des loyers, l'autre tiers des droits seigneuriaux. Des princes, comme le duc d'Orléans et d'autres grands nobles, vendirent des offices à ceux qui étaient prêts à faire respecter les droits seigneuriaux des grands de la cour pour leur propre bénéfice. Ces offices pouvaient compter jusqu'à 400 clients des maisons de la haute noblesse 9 .

A l'automne 1787, l'assemblée provinciale d'Orléans écrivit au ministère que, pour répartir les impôts d'une manière équitable, l'on ne pouvait faire confiance aux officiers municipaux d'Orléans et de Chartres, qui étaient désignés par les princes apanagistes. Le lieutenant de la Touchey, de la municipalité d'Orléans, répondit à cette accusation dans une lettre au contrôleur général en 1788 . Touchey écrivit que la ville appartenait à l'apanage du duc d'Orléans, qu'elle avait un statut juridique singulier et qu'elle devait être exemptée du nouvel impôt prélevé sous l'égide des assemblées et destiné à remplacer la corvée fournie obligatoirement par les paysans pour la construction et la réparation des chemins. La ville - soutint Touchey - payait traditionnellement les impôts indirects au lieu de la taille et, ainsi, ne devait pas être soumise à la nouvelle taxe avec les roturiers. Le roi décida alors d'approuver une proposition de l'assemblée permettant l'élection d'un conseil par les résidents payant 25 livres de taxe, pour siéger avec les anciens officiers du prince et pour répartir les impôts avec eux ${ }^{10}$.

L'assemblée du district de Laon consacra plusieurs séances à la justice fiscale et à la réforme des vingtièmes en 1788 et 1789 . Les membres remarquèrent que les vérifications de revenu couvraient 129 des 351 paroisses et avaient augmenté le rendement des vingtièmes d'un quart entre 1776 et 1784 . Le contrôleur des vingtièmes signala à l'assemblée que les propriétés toujours non vérifiées appartenaient au roi, aux princes, aux hôpitaux et à l'ordre de Malte. Ceux-ci ne fournissaient pas les renseignements nécessaires pour rendre les vérifications possibles. Le duc d'Orléans en particulier exprimait de la répugnance pour les vérifications et les défiait fermement ${ }^{11}$.

Dans le Lyonnais, le roi possédait plusieurs fiefs, et le duc d'Orléans était proprétaire de $25 \%$ de ceux du Beaujolais, y compris le droit d'administrer les corporations de métiers de Villefranche, la plus grande ville de ce pays. En 1786, le roi céda les droits seigneuriaux de plusieurs paroisses à l'ouest de Saint-Étienne au marquis d'Osmond, d'une famille au service du duc d'Orléans depuis des siècles et résidant au Palais Royal à Paris. Osmond s'engagea à appliquer ses droits et à interdire aux habitants de ramasser du charbon. Il adressa une pétition à l'intendant dans le but d'être le seul autorisé à en transporter au port de Saint-Just sur la Loire. Consultée sur l'affaire, l'assemblée du district de SaintÉtienne - composée, comme l'assemblée provinciale, de nobles éminents, de magistrats et de gros propriétaires, dont le plus grand nombre bénéficiaient de privilèges, $\mathrm{y}$ compris ceux qui siègeaient dans le tiers état - émit l'opinion que la concession d'Osmond était nuisible à la ville, à la province et aux habitants. D'autres seigneurs du pays virent aussi leurs privilèges miniers menacés par la concession d'Osmond ${ }^{12}$. 
Dans le cas précédent, les droits seigneuriaux divisaient les seigneurs de la cour et ceux du pays, mais en général ils établirent des liens, autour de traits distinctifs d'un certain standing, et encouragèrent les bourgeois à les rechercher. Les archives de la chambre domaniale de Montbrison montrent que des notaires, des officiers vénaux et d'autres bourgeois louaient les droits seigneuriaux du roi et des nobles et recherchaient souvent à en accroître la valeur en renouvelant les terriers. Les droits seigneuriaux, les lods et ventes et les banalités du Lyonnais étaient relativement lourds, atteignant jusqu'au tiers des vendanges, et comprenaient des travaux forcés pour la construction et la réparation des châteaux, le creusage des fossés et les guets ${ }^{13}$.

Le régime seigneurial, selon Serge Dontenwill et Christianne Lombard-Déaux, entraînait l'élite lyonnaise dans une hiérarchie d'autorités s'étendant de Versailles jusqu'aux châtelains provinciaux. Il constitua l'infrastructure d'une société reliée au passé féodal. Lors des assises organisées par les juges seigneuriaux de la région, ceux-ci procédèrent au dénombrement des justiciables, à la condamnation des absents à une amende, et aux serments de loyauté envers le roi et de soumission au tribunal du châtelain. Ces cours formaient un des points principaux de contact entre les autorités de l'Ancien régime et les sujets du roi. Selon C. Lombard-Déaux, les droits seigneuriaux délimitèrent les classes sociales du Lyonnais dans le sens où ils assuraient une liberté relative aux possesseurs, par rapport à ceux qui étaient subordonnés à leur cadre restrictif, désagréable et irrationnel ${ }^{14}$.

Les droits seigneuriaux entamèrent le succès des assemblées du Lyonnais. Les membres de l'assemblée provinciale et ceux des assemblées de district n'appréciaient pas les conseils élus dans les paroisses et se plaignaient des règlements. Selon ces derniers, le syndic élu par les villageois devait prendre la présidence de la paroisse si le seigneur n'était pas capable d'y assister. L'assemblée provinciale remarqua que plusieurs seigneurs possédaient les droits sur plus d'une paroisse et ne pouvaient pas assister à tous leurs conseils ruraux. Selon le procès-verbal de l'assemblée, ces réunions présidées par un syndic villageois seraient «tumultueuses et irrégulières, ce qui arrive assez ordinairement lorsqu'une assemblée composée de gens de la campagne n'a pas à sa tête quelqu'un qui inspire le respect et la confiance, qui les ramène à l'esprit de raison et [...] qui les contient par la supériorité de son rang $»^{15}$. Les habitants d'Ambierle essayèrent de changer le procès-verbal de leur conseil afin de remplacer leur syndic, par ailleurs procureur judicaire du seigneur. Ils écrivirent à l'assemblée provinciale que l'on ne pouvait pas être l'homme du seigneur et de la paroisse en même temps. Mais l'assemblée attira leur attention sur l'interdiction de remplacer les syndics ${ }^{16}$.

En 1776, le roi constitua pour le comte d'Artois le Berry en apanage, une donation de terres et de seigneuries, et l'accès à une masse de clients. Les officiers berrichons du comte d'Artois firent respecter ses droits seigneuriaux pour leur propre bénéfice, y compris ses droits exclusifs de chasse. Le duc de Béthune-Charost, un représentant du comte d'Artois et membre de l'assemblée provinciale formée en 1778, vendit sa seigneurie de Saint-Amand-Montrond, une ville d'environ 5000 habitants et une des principales du Berry, au comte François-Marie de Fougières, le premier maitre d'hôtel du comte d'Artois en 1778. Trois ans plus tard, les habitants écrivirent au contrôleur général à propos des abus dans les listes fiscales de la municipalité. Les personnages riches et réputés, ceux qui avaient de l'influence sur les percepteurs, s'étaient fait exempter des impositions municipales dont les autres propriétaires devaient suppléer la différence. L'assemblée provinciale écrivit au contrôleur général que l'on ne pouvait pas compter sur les officiers 
nommés par Fougières, un seigneur intéressé à la répartition des impôts, pour composer équitablement les listes fiscales. Le roi confia ensuite à l'assemblée provinciale et à l'élection de Saint-Amand (le tribunal du pays) la responsabilité d'assurer aux habitants la nomination de leurs propres percepteurs et un processus transparent pour la confection des listes fiscales. Mais les problèmes fiscaux demeurèrent, tout comme les mêmes personnagesinfluents continuèrent à tirer profit du ménagement des tribunaux et de l'administration, et tout comme l'assemblée se fia au comte de Fougières pour l'élaboration des listes fiscales ${ }^{17}$.

Vers la fin de 1779, l'assemblée du Berry accepta de faire un versement unique au roi - un abonnement - pour se réserver le droit de gérer elle-même les vingtièmes. L'assemblée supprima les dix contrôleurs qui avaient vérifié les revenus fonciers des privilégiés et qui avaient rapporté 140000 livres supplémentaires aux vingtièmes du Berry. Elle prononça que cette suppression susciterait du zèle parmi les contribuables et améliorerait la répartition. Les listes fiscales, énonça l'assemblée, devaient être faites par un nombre de trois à cinq assesseurs, nommés par des assemblées paroissiales composées de propriétaires. Les seigneurs possesseurs de biens dans plusieurs paroisses devaient faire les déclarations de revenus à l'assemblée afin qu'elle réduise les quotas des paroisses dans lesquelles se trouvaient leurs propriétés. Mais après avoir reçu une requête de l'intendant des finances du comte d'Artois, les membres de l'assemblée décidèrent de ne pas imposer ses propriétés dans le Berry et d'en prévenir les paroisses dans lesquelles se trouvaient ses domaines. En 1783, il n'y avait que 230 des 723 paroisses du Berry qui avaient nommé des assesseurs et composé les listes fiscales. Selon les plaintes des paroisses reçues par l'assemblée, les propriétaires aisés se servaient de leur influence pour ne pas respecter les réglements fiscaux. Seuls $23 \%$ des seigneurs avaient fait leurs déclarations, dont beaucoup, de surcroît, étaient incomplètes et inutilisables ${ }^{18}$.

Six ans plus tard, en 1787, le comte d'Artois et sa clique à la cour, ayant reçu des avantages financiers du contrôleur général, s'étaient ralliés au ministère et étaient favorables à l'ensemble des réformes de Calonne, y compris les assemblées provinciales, même si Artois était l'un des défenseurs de l'Ancien Régime les plus en vue. Toutefois, la maison du comte d'Artois embrassait des centaines d'officiers dont quelques uns, au moins, se voyaient lésés par les assemblées. Un mémoire anonyme, écrit au contrôleur général à propos des droits seigneuriaux d'Artois, objecta qu'il avait des seigneuries nombreuses et immenses et ne pouvait pas assister à tous les conseils paroissiaux devant avoir lieu dans ses possessions selon l'édit portant création des assemblées. Ses représentants n'assisteraient pas non plus pour la simple raison que, sans véritable titre de seigneur, ils n'avaient pas le droit à la présidence et refuseraient des rangs inférieurs au syndic paroissial. Il était indigne, suivant le mémoire, de siéger en-dessous de syndics que ces représentants embauchaient pour de vils travaux et des gages modiques. L'auteur du mémoire se plaignait des règlements qui avaient interdit aux représentants du comte d'Artois d'assister aux assemblées de district à cause du manque de titres de noblesse et de richesse requise. De plus, les princes possédaient des droits féodaux de mouvance, terrage et minage, ainsi que des droits aux étangs de pêche, bois, péages et autres. A l'inverse, d'autres seigneurs, ceux qui prenaient part aux assemblées provinciales, ne possédaient pas ces droits et avaient un intérêt dans l'imposition des propriétés féodales des princes sans rapport avec leurs propres affaires. Pour toutes ces raisons, l'auteur demandait que les représentantsdes princes aient la préséance dans les conseils paroissiaux et que les princes aient la présidence des assemblées provinciales. Selon le 
mémoire, ces mesures maintiendraient les bons rapports entre les princes et le roi, conserveraient leur gloire et mettraient le régime dans une lumière avantageuse ${ }^{19}$.

Après la famille royale, les principaux tenants des droits seigneuriaux étaient les grands nobles de Versailles. Le duc de Nivernais, par exemple, était membre de l'Académie française et donc probablement participait à la vie intellectuelle de l'époque. En 1780, lors du débat sur les assemblées du Berry et de la Haute-Guyenne, il écrivit un manuscrit au procureur général du parlement contre l'homologation du pouvoir administratif aux assemblées. Nivernais opéra un revirement en juin 1787 et se prononça en faveur des assemblées dans les débats du Parlement, bien qu'il vota ensuite contre le doublement du tiers état en tant que ministre sans portefeuille dans les conseils du roi en $1788^{20}$.

Le duc avait des domaines et des seigneuries dans le Nivernais rapportant plus de 300000 livres par an, pour lesquels il ne payait que 4000 livres au titre des vingtièmes. Il objecta, dans les lettres qu'il échangea avec le contrôleur général en 1787, qu'une seule assemblée de la généralité de Moulins, englobant le Nivernais et le Bourbonnais, serait trop grande et comporterait des propriétaires du Bourbonnais sans intérêt à entretenir avec lui des relations favorables. Nivernais n'aurait pas de facilités pour placer ses créatures et l'assemblée pourrait augmenter son imposition. Cet échange de lettres a certainement entrainé le roi à attendre jusqu'au milieu de 1788 pour créer les assemblées dans la généralité et la diviser en deux assemblées. L'assemblée plus restreinte du Nivernais permit au duc de placer ses vassaux et de continuer de sous-évaluer ses possessions. Par la suite, cette assemblée du Nivernais se montra réticente à l'égard de la représentation politique. Elle pétitionna auprès du contrôleur général pour restreindre la participation dans les réunions électorales programmées dans les années à venir afin de renouveler les assemblées de district ${ }^{21}$.

Somme toute, les grands de la cour contrecarrèrent les efforts des réformateurs royaux visant à introduire une mesure d'égalité dans la répartition de l'impôt. Il est vrai que, dans plusieurs cas, les assemblées procurèrent une occasion aux propriétaires provinciaux de se soutraire à la tutelle des grands et d'acquérir des responsabilités politiques, pourvu qu'ils collaborent avec le ministère. Ce phénomène s'est observé à Orléans, dans le Laonnais, et dans le Forez, où les privilégiés du pays, grâce aux assemblées, obtinrent une compétence administrative et virent de près les problèmes posés par les privilèges. Guy Chaussinand-Nogaret a insisté sur ce type de division et de conflit au sein de la noblesse, entre les grands et très riches de la cour d'un côté et les nobles aisés des provinces de l'autre. Dans un certain sens, l'usage que firent le roi et la noblesse des droits seigneuriaux élargirent cette division dans le Forez et à Saint-Amand, dans le Berry, où l'affirmation des privilèges, soutenue à Versailles, lésa les intérêts des propriétaires provinciaux ${ }^{22}$.

Mais au lieu de voir de la division et du conflit dans ces occurrences, j'affirme qu'on devrait plutôt y voir une direction par l'exemple. Les nobles de la cour, par l'usage qu'ils firent des clients régionaux - comme on le voit dans le Berry - pour éviter les vérifications des contrôleurs du vingtième, contribuèrent aux efforts des propriétaires provinciaux pour maintenir leurs exemptions et par conséquent parvenir à l'échec de l'objectif fiscal des assemblées. Le roi, sa famille et les grands nobles montrèrent l'usage que les détenteurs des droits féodaux pouvaient en faire et, dans le Lyonnais comme dans d'autres provinces, on voit bien que des détenteurs mirent en valeur méthodiquement leurs seigneuries afin d'acquérir du pouvoir et $\mathrm{du}$ profit $^{23}$. En érigeant des apanages pour 
sa famille et en cédant des seigneuries aux clients, le roi conservait la pertinence des lois féodales.

Les réformateurs royaux, tels que du Pont de Nemours et Necker, croyaient qu'une mesure de représentation politique permettrait aux administrateurs de surmonter les privilèges traditionnels et d'augmenter les impôts des nobles. Mais cette jurisprudence féodale, soutenue par la monarchie et appliquée par des seigneurs, fit obstacle à la représentation politique des ruraux et contribua ainsi à l'échec du but principal des assemblées.

\section{NOTES}

1. Alexis de TocqueVILLE, L'Ancien régime et la Révolution, Paris, Lévy, 1856 ; rééd. 1960, p. 256-264.

2. Pierre RENOUVIN, Les assemblées provinciales de 1787. Origines, développemment, résultants, Paris, A. Picard et J. Gabalda, 1921, p. 56-58, 60, 72-73, 78-80, 88 ; Maurice BORDES, « La ville de Lectoure, Pays d'Etats et l'Assemblée Provinciale d'Auch», Bulletin de la Société archéologique, historique, litteraire \& scientifique du Gers, 72, 1971, p. 537, 539; du même, L'administration provinciale et municipale en France au XVIII siècle, Paris, SEDES, 1972, p. 169-170; du même, "L'échec d'une décentralisation : les assemblées provinciales de 1787 ", L'information historique, 46, 1984, p. 222.

3. Peter JONES, «The Provincial Assemblies, 1778-1790; Old or New? ", Proceedings. Consortium on Revolutionary Europe, 1750-1850, 23, 1993-1994, p. 383-385 ; Peter JONES, Reform and Revolution in France. The Politics of Transition, 1774-1791, Cambridge, CUP, 1995, p. 8-9, 60, 114, 120-124, 144, 146, 155-159, 240.

4. William BEIK, Absolutism and Society in Seventeenth-Century France. State Power and Provincial Aristocracy in Languedoc, Cambridge, CUP, 1985, 375 p. ; Robert DESCIMON et Christian JOUHAUD, La France du premier XVIIe siècle, 1594-1661, Paris, Belin, 1996, 233 p. ; Marie-Laure LEGAY, Les états provinciaux dans la construction de l'état moderne, aux XVII et XVIII siècles, Genève, Droz, 2001, 565 p. Les autres œuvres qui soutiennent cette thèse sont: Daniel DESSERT, Argent, pouvoir et société au Grand Siècle, Paris, Fayard, 1984, 824 p. ; Pierre GOUBERT et Daniel ROCHE, Les Français et l'Ancien Régime, Paris, A. Colin, 1984, 2 vol. ; Herbert LÜTHY, La banque protestante en France, de la révocation de l'Édit de Nantes à la Révolution, Paris, SEVPEN, 1959, 454 p.; Julian SWANN, Provincial Power and Absolute Monarchy. The Estates General of Burgundy, 1661-1790, Cambridge, CUP, 2003, $460 \mathrm{p}$.

5. Pierre DU PONT DE NEMOURS, « Mémoire sur les municipalités ", Oeuvres de Turgot et documents le concernant, Paris, F. Alcan, 1922, t. 4, p. 574-577, 580-582.

6. Jacques NECKER, «Extrait du mémoire de M. Necker, présenté au roi en 1778 sur l'établissement des administrations provinciales », Objets proposés à l'Assemblée des notables par de zélés citoyens, Paris, imprimerie Polytype, rue Favart, 1787, p. 27, 29, 33.

7. Jacques NECKER, De l'administration des finances de la France, Paris, Panckoucke, 1784, t.1, p. 122-123, 160.

8. David PARKER, «Absolutism, Feudalism and Property Rights in the France of Louis XIV », Past and Present, 179, 2003, p. 91-92; Thomas KAISER, «Property, Sovereignty, the Declaration of the Rights of Man, and the Tradition of French Jurisprudence », The French Idea of Freedom. The old Regime and the Declaration of Rights of 1789, Stanford (Calif.), Stanford University Press, 1994, p. 309-310, 312-315. 
9. Pierre GOUBERT, "La société traditionelle ", in Histoire économique et sociale de la France, dir. Fernand Braudel et Ernest Labrousse, Paris, PUF, 1970, t. 2 p. 584 ; Mathieu MARRAUD, La noblesse de Paris au XVIII siècle, Paris, Le Seuil, 2000, p. 122, 240-241, 308-309.

10. Arch. Nat. $\mathrm{H}^{1} 590$; Henry FROMONT, Essai sur l'administration de l'assemblée provinciale de la généralité d'Orléans (1787-1790), Orléans, H. Houzé, 1907, p. 17-18, 22.

11. Cécile souchon, "L'assemblée d'élection de Laon et les 'affaires du pays'", Les pouvoirs régionaux, représentants et élus, Paris, CTHS, 1987, p. 112-113.

12. Arch. Dép. Loire C 76 ; Arch. Dép. Rhône, 9C 73 ; Paul TÉzenAS du montCEL, Étude sur les assemblées provinciales du département de Saint-Étienne et sa commission intermédiaire (8 octobre 1787 21 juillet 1790), Paris, H. Champion, 1903, p. 249-250, 255, 260 ; Procès-verbal des séances de la première assemblée provinciale de la généralité de Lyon, tenue à Lyon, dans les mois de septembre, novembre \& décembre 1787, Lyon, 1787, p. 2-3, 8-11; Jacques RIVOIRE, Soucieu-en-Jarez et ses environs. Étude historique, folklore régional, de Mornant à Vaugneray, Lyon, Bost frères, 1979, p. 40, 44 ; JeanBaptiste GALLEY, L'élection de Saint-Étienne à la fin de l'ancien régime, Saint-Étienne, 1903, p. 368-369 ; du même, Saint-Etienne et son district pendant la Révolution, Saint-Etienne, 1903-1907, p. 21 ; Louis TRÉNARD, «La crise sociale lyonnaise à la veille de la Révolution ", Revue d'histoire moderne et contemporaine, 1955, p. 17 ; Mathieu MARRAUD, La noblesse ... op. cit., p. 242.

13. Arch. Dép. Rhône, 9 C 72, C 236 ; Josette GARNIER, Bourgeoisie et propriété immobilière en Forez aux $\mathrm{XVII}^{e}$ et XVIII ${ }^{e}$ siècles, Saint-Etienne, Centre d'études foréziennes, 1982, p. 161-162 ; Christianne LOMBARD-DÉAUX, Seigneurs et seigneuries en Lyonnais et Beaujolais des guerres de religion à la Révolution. Organisation, fonctionnement, évolution de la vie des campagnes, Lyon, éd. Bellier, 2005, p. 76, 129 ; Serge DONTENWILL, «Le rôle des assises et réglements de seigneurie dans la régulation sociale aux $\mathrm{XVII}^{\mathrm{e}}$ et XVIII ${ }^{\mathrm{e}}$ siècles. Le cas du Centre-Est de la France ", Les justices de village. Administration et justice locales de la fin du Moyen Age à la révolution, actes du colloque d'Angers des 26 et 27 octobre 2001, Rennes, PUR, 2002, p. 231 ; Louis VIGNON, Annales d'un village de France, Charly-Vernaison en Lyonnais, Charly, chez l'auteur, 1978, t. 3, p. 417 ; Edouard BRENOT, Documents pour servir l'histoire de Grignyen-Lyonnais des origines à 1789, Grigny, CEuvre municipale pour l'enfance et la jeunesse, 1980, p. 94. 14. Arch. Dép. Rhône, 3 B (ct6905) ; René LAPLACE, L'histoire d'Oullins des origines à 1900, Lyon, éd. L'Hermès, 1977, p. 30 ; Christianne LOMBARD-DÉAUX, Seigneurs et seigneuries...op. cit., p. 143 ; Serge DONTENWILL, «Le rôle des assises... art. cit. », p. 224-226; Christian LAURANSon, "Les justices seigneuriales en Forez à la fin de l'ancien Régime ", Bulletin du Centre de Recherches Historiques de l'Université de Saint-Etienne, 1988-1989, p. 56-57.

15. Paul TÉZENAS DU MONTCEL, Étude sur les assemblées provinciales... op. cit., p. 153.

16. Ibid., p. 292 ; Arch. Dép. Rhône, 9C 13 ; Etienne BROSSARD, Histoire du département de la Loire pendant la revolution française (1789-1799), publié par Joseph de La Poix de Fréminvielle, SaintÉtienne, Chevalier, 1904-1907, p. 20.

17. Arch. Dép. Cher, B 4307, C 231, C 1090, C 1114, C 1142, C 1315, E 205, B 4307 ; Anne-Marie AUBIN, Criminalité en Berry au XVIII siècle, Paris, Royer, 2001, p. 141 ; Maire-Camille, vicomte THIERRY DE BRIMONT, M. de Puységur et l'église de Bourges pendant la révolution, 1789-1802, Bourges, Tardy-Pigelet, 1896, p.44-45; Fernand DUMONTEIL, Une ville seigneuriale en 1789, Saint-AmandMontrond, Bourges, H. Sire, 1887, p. 52, 59-60.

18. Arch. Dép. Cher C 1315; Marcel MARION, L'impôt sur le revenu au dix-huitième siècle, principalement en Guyenne, Toulouse, Privat, 1901; rééd. Genève, Slatkine, 1976, p. 224-226 ; Augustin RIOCHE, De l'administration des vingtièmes sous l'ancien régime, Paris, Rousseau, 1904, p. 121-122.

19. Arch. Nat. $\mathrm{H}^{1} 519$; Munro PRICE, Preserving the Monarchy. The Comte de Vergennes, 1774-1787, Cambridge, CUP, 1995, p. 28-29, 152-153 ; du même, The Fall of the French Monarchy. Louis XVI, Marie Antoinette and the baron de Breteuil, New York, 2003, p. 6-7, 59. 
20. Bibliothèque Nationale, Collection Joly de Fleury, 1037 ; Mathieu MARRAUD, La noblesse... op. cit., p. 409 ; Joël fÉLIX, Louis XVI et Marie-Antoinette. Un couple en politique, Paris, Payot et Rivages, 2006, p. 451.

21. Arch. Nat. $\mathrm{H}^{1} 603, \mathrm{H}^{1} 593$; Les États Provinciaux comparés aux Assemblées provinciales, p. 33 en note.

22. Guy CHAUSSINAND-NOGARET, La noblesse au XVIII siècle.De la féodalité aux lumières, Paris, éd. Complexe, 1976, p. 17, 77, 86.

23. Gérard AUBIN, La seigneurie en Bordelais au XVIII ${ }^{e}$ siècle d'après la pratique notariale, 1715-1789, Rouen, Publications de l'Université de Rouen, 1989, p. 180, 381, 388, 394, 400-401, 446-447, 449 ; Guy lemarchand, La fin du féodalisme dans le pays de Caux, Paris, éd. du CTHS, 1989, p. 21, 294-295, 313-315, 328-329 ; Peter MCPHEE, Revolution and Environment in Southern France. Peasants, Lords and Murder in the Corbières, Oxford, Clarendon Press, 1999, p. 26-28, 33-34 ; Robert FORSTER, The House of Saulx-Tavanes, Versailles and Burgundy, 1700-1830, Baltimore, John Hopkins Press, 1971, p. 92-104 ; Peter JONES, Politics and Rural Society. The Southern Massif Central, c. 1750-1880, Cambridge, CUP, 1985, p. 163 ; Florence GAUTHIER, La voie paysanne dans la Révolution française. L'exemple de la Picardie, Paris, F. Maspero, 1977, p. 80-81, 83.

INDEX

Mots-clés : assemblées politiques, monarchie, féodalité

Index chronologique : XVIIIe siècle

Index géographique : Berry, Haute-Guyenne, Lyonnais, Nivernais, Bourbonnais

\section{AUTEUR}

\section{STEPHEN MILLER}

Associate Professor, Department of History, University of Alabama at Birmingham, 1401 University Blvd., HHB 360Q, Birmingham, AL 35294-1152 